the cases fulfilled the criteria of sarcoidosis as defined by Scadding, ${ }^{2}$ the majority of patients did not present in the usual way with sarcoidosis, and indeed a survey of chest clinic and eye and skin department material has been unprofitable in revealing cases with myocardial involvement. These chiefly present with the cardiac problems of heart block, difficult tachydysrhythmias, frequent ventricular extrasystoles, sudden death, and congestive cardiac failures, in that order of frequency.

Twenty patients in the series have died and the diagnosis has been confirmed postmortem. In all, other organs were involved, often to a clinically undetectable degree. In many cases sarcoid involvement of the heart was massive, and in these the classical sarcoid granulomatous tissue with large numbers of giant cells was widespread. In less massive involvement the ventricular septum appears to be the site most commonly affected.

I am preparing the full results of this study for detailed publication. A preliminary report of over 40 cases was made to the Sixth European Congress of Cardiology in Madrid. ${ }^{3}$. It was there suggested that the diagnosis of sarcoidosis should be considered in any patient presenting with a cardiomyopathy of unknown aetiology, particularly if there are frequent ventricular premature systoles, or in any patient who presents with a serious rhythm disturbance of any type. The success of steroid treatment will depend on how massive is the sarcoid involvement of the heart.

This study has been made possible by the generous co-operation of colleagues allowing me access to their material. I would be glad to have details of any further cases.-I am, etc.,

Addenbrooke's Hospital,
Cambridge

Hugh A. Fleming

1 Ghosh, P., Fleming, H. A., Gresham, G. A., and Stovin, P. G. I., British Heart fournal, 2 Scadding, J. G., Sarcoidosis. London, Eyre and Spottiswoode, 1967.

Fleming, H. A., Revista Española de Cardiologia.

Proceedings of the 6th European Congress of Cardiology, Madrid. In press.

\section{Spin Dryer Injuries}

SIR,-Mr. C. S. B. Galasko (16 December, p. 646) rightly draws attention to the dangers associated with the use of spin dryers. I realize that the pictures of trauma vary from one part of the country to another. In my experience in the north-east of England, I have seen these injuries about 12 times a year in casualty departments receiving 40,000-50,000 new patients a year. These injuries are not confined to children and are replacing the "wringer injury." As with wringer injuries, soft-tissue damage is out of proportion to the bony damage; this is due to the tremendous rotational forces involved. Unlike wringer injuries, these are not virtually confined to the appliances made by one manufacturer.

Spin dryer injuries are related to the kinetic energy involved. It therefore follows that the large drums which mostly rotate on a horizontal axis tend to produce much more severe injuries than small drums, which generally rotate on a vertical axis, where the speed of revolution is similar. Horizontally rotating drums are more accessible to children.
The most common injuries affect the ingers-generally there is dislocation at the proximal interphalangeal joint. This may be inadequately treated by the casualty officer, for the fact that one or both collateral ligaments are torn may be overlooked. However, about one-third of these injuries are compound and it is, I hope, unlikely that an inexperienced casualty officer would be left to deal with these. On occasions, multiple digits may be dislocated.

Injuries at higher levels than the fingers are generally not associated with injuries to the hand, just as injuries to the humerus are unlikely to be associated with injuries below this level. I have seen seven spin dryer injuries to forearm bones, five of which occurred in children. It is again importan to realize that damage to soft tissues associated with these injuries is often much more extensive than an $x$-ray of the forearm would lead one to suppose. Ischaemic contracture and muscle fibrosis must be regarded as definite risks, and forearm decompression may have to be considered.

I have seen three fractures of the humerus from this cause. Once again, softtissue damage predominates, and it is not easy to identify the anatomy at the site of injury. I have been more fortunate than Mr. Galasko in that in my worst experience of this type of injury at this level all structures were divided apart from the neurovascular bundle containing the median and ulnar nerves. Progress in this child has therefore been quite good.

I must point out that all the spin dryers that I have seen do in fact have some device either to prevent the dryer being opened while in motion or to arrest the dryer when the lid is opened, though it may be possible to bypass the latter type of device. Injuries occur when the safety devices fail, failure of the former type resulting in more severe injury than failure of. the latter. It is not easy to suggest what further steps manufacturers could take. I would refer those interested to British Standard 3456 B6, which specifies that with the latter type of device the drum should stop spinning within 10 seconds of the lid being opened. This standard comes into force in 1974. Which? in March 1971 reported on the mechanical safety of these devices and found that with most makes the drum in fact stopped within five seconds of the lid being lifted.-I am, etc.

Middlesbrough General Hospital,
Teesside

PAUI R. J. VIckers

\section{Side Effects of Benorylate}

StR,-Benorylate is the esterification product of paracetamol and acetylsalicvlic acid. It has been shown to have fewer gastrointestinal side effects, including blood loss, than aspirin or an equimolecular mixture of aspirin and paracetamol ${ }^{2}$ while retaining their analgesic and anti-inflammatory properties. ${ }^{2}$ Recorded side effects have been nausea, constipation, indigestion, heartburn, drowsiness, tinnitus, dizziness, and skin rashes. Here we report the case of a patient who was unable to tolerate the drug because of diarrhoea.

A 67-year-old woman with a three-vear history of rheumatoid arthritis was admitted with severe active arthritis in her shoulders, elbows, wrists, and metacaroophalangeal. proximal interphalangeal, and knee joints. She was ound to be iron deficient; this was attributed to the salicylate therapy which she had been receiving almost continuously for two years.

She was treated with prednisolone at low dosage $(7.5 \mathrm{mg}$ daily), indomethacin suppositories, ferrous gluconate (Sidros), digoxin, pyridoxine, and $5 \mathrm{ml}$ of Benoral (containing 1 . benorylate) in suspension was given four times daily. At this dosage the patient noticed lower abdominal discomfort within five minutes of taking the benorylate, and the discomfort passed off after a further five or 10 minutes. After three days, because of joint pain between doses, the dosage of Benoral was increased to $8 \mathrm{ml}$ five imes daily. At this dosage the patient noticed lower abdominal colicky pain within 10 minutes of taking the benorylate, followed within five minutes by an urgent call to stool, the motion being very loose and on occasions watery. No blood was observed and faecal occult blood tests were negative. All drugs except the oral steroids and benorylate were stopped, but the symptoms persisted for two more days until the benorylate was discontinued, since when no further diarrhoea occurred.

We feel confident that the diarrhoea was related to the benorylate and, so far as we can determine, is a hitherto unrecorded side effect.

We are grateful to Dr. D. Mattingly for permission to publish this case.

-We are, etc.,

A. J. Marshall Peter Sheridan

Royal Devon and Exeter Hospital,

Exeter

Cuddigan, J. H. P., Abstracts of the VII European Rheumatology Congress, No. 45, 7 June pean

Lievre, J. A., Information Thérapeutique, 1969,
7,49.

\section{Treatment of Massive Pulmonary Embolism}

SIR,-The case reported by Dr. R. J. C. Hall and others (16 December, p. 647) is further evidence of the effectiveness of streptokinase in the treatment of massive pulmonary embolism.

The conclusion that the drug was successful in lysing clot in the pulmonary arterial tree was based on clinical assessment-the patient's general state, recovery from dyspnoea, lowering of central venous pressure and pulse rate, etc. While this is probably acceptable, the absence of precise objective evidence of thrombolysis, such as could have been supplied by further pulmonary angiography or lung scanning, prevents any accurate assessment of the effective dose of streptokinase and its rate of infusion. The authors used a 41-hour infusion with a total dose which we calculate to have been $4,650,000$ units. In our experience of nine cases of massive pulmonary embolism treated successfully with streptokinase-monitored before, during, and after streptokinase treatment by lung scanninga 12-hour infusion of $1,500,000$ units has been adequate to produce lysis of thrombus in the pulmonary artery. The present report states that within eight hours the patient was greatly improved. This suggests that lysis was advanced by that time. In this context it should be remembered that thrombolvsis will continue for periods uo to days after the cessation of streptokinase therapy. Furthermore, many of the dangers of streptokinase therapy, particularly in relation to fibrinogen rebound, exhaustion of plasminogen, and rethrombosis, are more likely to occur the longer the infusion lasts and, in fact, sometimes do occur even during pro- 